\title{
Identification of Genes in a Thyroid Cell Line Regulated by Thyroid-Stimulating Hormone (TSH)
}

\author{
Minho Shong ${ }^{\mathrm{a}}$, Yong-Jin Kim ${ }^{\mathrm{b}}$, Yongmun $\mathrm{Choi}^{\mathrm{b}}$ and O-Yu Kwon ${ }^{\mathrm{b}} *$ \\ a Department of Internal Medicine and \\ b Anatomy, College of Medicine, Chungnam National University, Taejon 301-131, Korea. \\ Fax: 82-42-580-8206, E-mail: oykwon@hanbat.chungnam.ac.kr \\ * Author for correspondence and reprint requests \\ Z. Naturforsch. 54c, 578-582 (1999); received March 8/May 4, 1999 \\ Differential Display (DD) PCR, Thyroid Stimulating Hormone (TSH), \\ Rat Thyrocytes (FRTL5 cells)
}

Differential display (DD) PCR (Liang and Pardee, 1992) is a recently described technique to identify genes whose expression has changed during a biological process. We used this method to detect genes thyroid stimulating hormone-dependently regulated in a rat thyroid cell line, because thyroid stimulating hormone (TSH) is the most important hormone for cell proliferation and differentiation including prehormonal proteins secretion in thyrocytes (Kim and Arvan, 1991; Kim and Arvan, 1993). Following DD-PCR experimentation, thyroid stimulating hormone -dependently regulated gene fragments of 15 species were obtained. The genes were used as molecular probes in Northern blot analysis and then sequenced. Two of the clones (\#123 and \#205) were up-regulated and two more (\#107 and \#111) were downregulated thyroid stimulating hormone-dependently in the thyroid cells, as demonstrated by Northern blot analysis. Following partial sequencing, each of the clones \#107, \#111 and \#205 were shown to be homologues of the apoptosis-related gene, aldolase A, and a-2 collagen (IV), respectively, while clone \#123 showed no homology with known genes. These findings suggest that the four genes mentioned above may have an a important physiological function in the thyrocytes, which is thyroid stimulating hormone-dependently up-/down-regulated.

\section{Introduction}

The human thyroid gland, located in the neck behind the larynx or voice box, helps to regulate most of the body's systems through the release of thyroid hormone. Hypothyroidism, with an incidence of approximately $1 / 40,000$ humans, is the most common health problem associated with the thyroid gland and causes underactive thyroid functioning. Patients may exhibit a range of phenotypes, including thyroid growth retardation, abnormal development of the central nervous system, and local compression of the neck tissues (Medeiros-Neto et al., 1993). Our previous data (Kim et al., 1996) have demonstrated that the unfolding or incorrect folding of thyroglobulin ( $\mathrm{Tg}$ ), associated with molecular chaperones in the endoplasmic reticulum (ER). However, we do not fully understand its molecular mechanism. It is well known that the thyroid hormone is dominantly regulated by TSH (Kim and Arvan, 1993). In order to understand how hypothyroidism is associated with a factor which is TSH-dependently regulated at molecular level we isolated TSH-dependently regu- lated genes using the differential display-PCR (DD-PCR) method from cultured rat thyroid cells (FRTL5 cells). In this paper, we report on the initial steps in understanding how partial sequences and mRNA expressions of the four genes are important factors in the regulation of $\mathrm{Tg}$ synthesis and secretion from the dysthyroid gland, and therefore, cause hypothyroidism.

\section{Materials and Methods}

\section{Cell culture and poly $(A)^{+} R N A$ isolation}

The rat thyroid cell line FRTL5 (Kohn and Valente, 1989) was cultured in Coon's medium containing $5 \%$ calf serum and $10^{-9} \mathrm{M} \mathrm{TSH}, 5 \mu \mathrm{g} / \mathrm{ml}$ transferrin, $1 \mu \mathrm{g} / \mathrm{ml}$ insulin, and $10 \mathrm{~nm}$ hydrocortisone at $37{ }^{\circ} \mathrm{C}$ and $5 \% \mathrm{CO}_{2}$ conditions until confluent. The resulting cells were washed three times with cold PBS to completely remove the hormones and then incubated for $72 \mathrm{~h}$ with or without TSH as the growth medium. The collected cells were transferred in a sterile microtube and mRNA was 
isolated following manufacturer's instructions Micro-FastTrack ${ }^{\mathrm{TM}}$ Kit (Invitrogen, Carlsbad, USA). The mRNA pellets were suspended in diethylpyrocabonate (DEPC)-treated water, and the yield was determined using a UV spectrophotometer or the DNA DipStick ${ }^{\mathrm{TM}}$ Kit (Invitrogen, Carlsbad, USA).

\section{Differential display PCR (DD-PCR)}

DD-PCR was performed according to the method of Liang and Pardee (1992), which enables researchers to detect a large number of mRNAs that are expressed in one RNA population but missing in another, and does not limitations of other methods such as subtractive hybridization and differential screening (Diachenko et al., 1996). Its basic principle is that the first step is reverse transcription against RNA as a template, using a primer for reverse transcription based on oligo (dT). After reverse transcription and denaturation, arbitrary priming is performed on the resulting strand of cDNA. PCR can now take place to generate a products that best matches the primers and that are derived from the $3^{\prime}$ end of the mRNAs and polyadenylated heterogenous RNAs. The resulting PCR products are separated on the SDS-PEGE with control's to detect differentially expressed RNA. Five micrograms of mRNA were incubated at $65^{\circ} \mathrm{C}$ for $15 \mathrm{~min}$ before reverse transcription. The resulting product was amplified using ten arbitrary primers -5'-ATTAACCCTCACTAAAN $_{9}-3^{\prime}$ and nine oligo (dT) PCR primers 5'-CATTATGCTGAGTGATATCT ${ }_{9} \mathrm{~N}_{2}{ }^{-3}{ }^{\prime}$ using a Delta ${ }^{\mathrm{TM}}$ Differential Display Kit (Clontech, Palo Alto, USA) in the Perkin-Elmer DNA machine. Each pair of samples was subjected to 25 cycles at $94{ }^{\circ} \mathrm{C}$ for $1 \mathrm{~min}, 60^{\circ} \mathrm{C}$ for $1 \mathrm{~min}$, and $68^{\circ} \mathrm{C}$ for $2 \mathrm{~min}$; the last cycle was followed by a 7 min extension at $68^{\circ} \mathrm{C}$. The amplification products were denatured at $80{ }^{\circ} \mathrm{C}$ on a heating block for $2 \mathrm{~min}$, and separated on a $5 \%$ sequencing gel; the dried gel on a Whatman filter paper was exposed to X-ray film. The potential differentially regulated cDNAs were eluted by boiling and ligated in the pGEM-T Easy vector $(3,018 \mathrm{bp}$ ) (Promega, Madison, USA). Inserted genes were checked on the agarose gel after digestion with EcoRI.

\section{Northern blotting analysis}

The mRNA expression level of the genes was analyzed by Northern blotting. Total RNA from the two cells, grown with and without TSH, was prepared by a Micro-FastTrack ${ }^{\mathrm{TM}}$ Kit (Invitrogen, Carlsbad, USA). Equal amounts of RNA $(20 \mu \mathrm{g})$ were separated on a denaturing agarose gel $(2.5 \%$ agarose containing $2.2 \mathrm{M}$ formaldehyde) and transferred to a nylon membrane (Boehringer Mannheim, Mannheim, Germany). After the filter was UV-crosslinked (UV-stratalinker 1800) (Stratagene, La Jolla, USA), it was then prehybridized in a high SDS buffer [7\% SDS, 50\% formamide, $5 \times$ SSC (sodium citrate $43.83 \mathrm{~g}$ and sodium chloride $22.06 \mathrm{~g}$ in 1 liter $\mathrm{H}_{2} \mathrm{O}$ )], $2 \%$ blocking reagent, $50 \mathrm{~mm}$ sodium phosphate] at $50{ }^{\circ} \mathrm{C}$ for $2 \mathrm{~h}$. Hybridization was performed with the same SDS buffer containing $\left[{ }^{32} \mathrm{P}\right]$-dATP leveled gene fragments at $50{ }^{\circ} \mathrm{C}$ overnight. The nylon membrane was rinsed twice with $2 \times \mathrm{SSC}$ and $0.1 \%$ SDS at room temperature. The membrane was then exposed to an Xray film in a deep-freeze for between $5 \mathrm{~h}$ and 1 day to allow the development of mRNA signals. Gene fragments were isolated from the agarose gel by a silica-based matrix of DNA PreMate ${ }^{\mathrm{TM}}$ Kit (Bioneer, Taejon, Koera), and were used as molecular probes for Northern blot analysis.

\section{Nucleotide sequencing}

In order to determine the partial sequences of genes, a Wizard minipreps DNA purification Kit (Promega, Madison, USA) was used to isolate the plasmids. Automatic nucleotide sequencing was performed by the standard dideoxy chain termination method on double-stranded plasmid DNA using the DNA-sequencing Kit (U. S. Biochemical, Cleveland, USA). Computer analyses of the nucleotide was performed using the BLAST sequence searching program of the National Center for Biotechnology Information, Bethesda, U. S. A.

\section{Results}

We employed the DD-PCR method to screen genes that are differentially regulated by TSH in FRTL5 cells. mRNAs from cells grown with or without TSH were reverse transcribed onto cDNAs using oligo-dT primers. Selected portions were amplified by PCR using one cDNA primer 
and the other $5^{\prime}$ arbitrary primers. The resulting PCR products were separated on a $5 \%$ sequencing gel. Fifteen species of cDNA fragments TSH-dependently expressed in FRTL5, were isolated using DD-PCR. They were subcloned onto the pGEM-T Easy vector (Promega, Madison, USA) and EcoR I digested to estimate the size of inserted DNA; the results are shown in Table I. The inserted DNAs ranged in size from 150 to 600bp.

A total of 15 species of cDNA fragments, isolated from cell grown with and without TSH, were used as probes for Northern blotting against total RNAs, to confirm that these clones are expressed differently by TSH. The results of expression of these clones are shown in Fig. 1. Four clones out of 15 DNA bands were confirmed to be differentially up-/down-regulated by TSH. As shown in Fig. 1, two clones, \#123 and \#205, were differentially up-regulated in TSH-grown cells, while the other two clones, \#107 and \#111, were highly expressed in cells grown without TSH. However, the other 11 of the 15 clones showed little or no differential expression in FRTL5 cells (data not shown).

The four previously mentioned cDNAs were partially sequenced and, when compared to those known DNA sequences found on the GenBank data bases, three of the clones (\#107, \#111 and \#205) were homologous with already reported genes, while the fourth clone (\#123) had novel DNA sequence. Table I shows the data regarding the sequencing analysis of the four clones. Clone \#123 does not share any meaningful homology with previously documented genes, while clones \#107, \#111, and \#205 are closely matched to the apoptosis-related gene (Onishi and Kizaki, 1996), aldolase A (Mukai et al., 1986), and a-2 collagen IV (Kurkinen et al., 1987), respectively, with more than $90 \%$ DNA sequence homology.
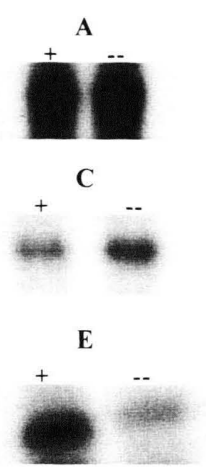

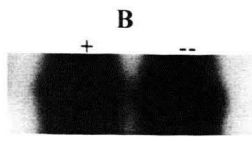

D

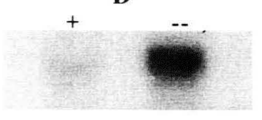

F

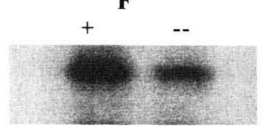

Fig. 1. Northern blot analysis of mRNA from FRTL5 cells.

Approximately $20 \mathrm{mg}$ of total RNAs isolated from FRTL5 cells (indicated by + ) and without TSH (indicated by -) were used for Northern blot analysis. The molecular probes are shown in the different panels-A and $\mathrm{B}$ : the controls, represented by fragments of $18 \mathrm{~S}$ and 28S rRNA genes, C: clone \#107 (apoptosis-related gene); D: clone \#111 (aldolase A); E: \#123 (novel gene); F: \#205 $(a-2$ collagen IV).

\section{Discussion}

We employed the DD-PCR method to screen genes that are differentially regulated by TSH in FRTL5 cells. FRTL5 cells were cultured on Coon's medium (Kohn and Valente, 1989) with and without TSH in a 6 -well plate until $100 \%$ confluence. Although two distinct cell types were used, microscopy showed that they both had some similar basic morphological characteristics: however, the growth rate of $\mathrm{TSH}$-grown cells was relatively faster than that of cells grown without TSH grown. Reverse-transcribed cDNA pools from mRNAs, isolated from both cell types, were used as templates in the PCR performed using a combination of forward and reverse primers with $\left[{ }^{32} \mathrm{P}\right]$-dATP. The amplified PCR products from both cDNA pools were compared on a $5 \%$ denatured acrylam-

Table I. Isolation of TSH-dependently regulated gene fragments in FRTL5 cells.

\begin{tabular}{llcccc}
\hline Clone & No. & Homology & Percentage ID & Species & NB \\
\hline$\# 107$ & D50465 & $64 / 69$ & $(92)$ & mouse & $(-)$ \\
$\# 111$ & X04262 & $238 / 251$ & $(94)$ & rat & $(-)$ \\
$\# 123$ & novel & - & - & - & $(+)$ \\
$\# 205$ & X04647 & $285 / 300$ & $(95)$ & mouse & $(+)$ \\
\hline
\end{tabular}

\#107 (apoptosis-related gene), \#111 (aldolase A), \#123 (novel gene), and \#205 ( $a$-2 collagen IV). No: accession numbers of the matched sequences. Homology: number of identical sequences for nucleotide matches (query/subject). Percentage ID: percentage identity. NB: Result of Northern blot analysis, +: up-regulated by TSH; -: down regulated by $\mathrm{TSH}$. 
ide gel. The PCR products originating from both pools exhibited largely similar pattern on the Xray film, but $15 \mathrm{cDNA}$ bands were uniquely expressed either in the cells grown with or without TSH. Unique DNA fragments were isolated from the dried gel and reamplified by PCR. These were then inserted into the multicloning sites of the pGEM-T Easy vector which has EcoR I sites at both multicloning regions and contain $\mathrm{T}$-tail to allow efficient ligation of the PCR product. To confirm the length of inserted DNA fragments, plasmids prepared by the alkaline lysis method from white or blue colonies were digested with EcoRI and separated onto the agarose gel. Because some inserted DNA fragments in this plasmid were too short less than $150 \mathrm{bp}$, their color screening on the ampicillin-LB medium containing $\mathrm{X}$-gal and IPTG, was not easy. Some blue colored clones contained inserted DNA fragment in contrast to some white colored clones, which contained no DNA fragments. Based on this result, we recommend that cDNA band lengths should exceed 150bp in order to detect positive DNA fragments using the DD-PCR method. Each inserted DNA fragment was estimated to have been between approximately $50 \mathrm{bp}$ and $600 \mathrm{bp}$ in length.

To confirm TSH-dependently regulated unique cDNA clones, the mRNA expression was tested by Northern blot analysis. Fifteen clones hybridized with two types of total RNAs, were isolated from cells grown with or without TSH. While 11 clones showed no dominant expression in cells grown with or without TSH four of the 15 clones, \#107, \#111, \#123 and \#205, showed signs up-/downregulation by TSH (Fig. 1). Thus, TSH-differentially up-regulated clones \#123 and \#205, while it differentially down-regulated clones \#107 and \#111 in the FRTL5 cells. To determine the partial sequences information of the four clones above, the plasmids were prepared and subjected to the automatic sequencer. DNA sequences of clones $\# 107$, \#111 and \#205 closely matched the apoptosis related gene, aldolase $\mathrm{A}$, and a-2 collagen (IV), respectively. However, clone \#123 did not show any significant homology with known genes in the current NCBI database. These results are summarized in Table I.

Although their role is still not clear with regard to thyrocytes, based on the limited information acquired from other cells, we can conclude that
cDNA clones of aldolase A, the apoptosis-related gene, and $a-2$ collagen (IV) play a important roles by up-/down-regulation by TSH. In particular, we speculate on those roles associated with Tg synthesis and secretion. This is supported by the knowledge that in the case of the apoptosis-related gene, TSH enhanced $\mathrm{T}_{3}$ synthesis, which in turn down-regulates poly(ADP-ribose)polymerase whose function is to inhibit the topoisomerase activity of DNA unwinding, and also prevent DNA fragmentation associated with apoptosis (Giannoni et al., 1995; Shibahara et al., 1995). Although the aldolase A gene is the first target for the thyroid hormone receptor superfamily transcription, the activated receptors bind to responsive promoters and mediate the assembly of the transcriptional complex which may be inhibited by TSH maintaining the homeostasis of $\mathrm{Tg}$ synthesis (Chang et al., 1997). There is a large degree of overlap between the mRNA species induced by TSH and carbohydrates (Liu and Towle, 1994). Gomez et al. (1994) reported that insulin and dexamethasone were enhancers of aldolase gene expression, while TSH was suppressive. It is well known that TSH markedly stimulates FRTL5 cells proliferation and differentiation; however, little is known about the function of collagen species as an extracellular matrix in the thyrocytes (BurgiSaville et al., 1997). Ulisse et al. (1998) demonstrated that $T_{3}$ treatment significantly reduced collagen IV secretion and increased laminin and entactin in sertoli cells. In this respect, our result showing an association between $a-2$ collagen (IV) up-regulation and the proliferation and differentiation of thyrocytes is interesting, particularly in the case of clone \#123, which is a novel gene expressed dominantly by TSH. Further study is still required in order to ascertain the function of clone $\# 123$ and also the functions of clones \#107, \#111 and \#205, which are homologues of the apoptosisrelated gene, aldolase A, $a$ - 2 collagen (IV), respectively, with regard to their regulation of Tg folding, assembly and secretion and hence their role in hypothyroidism.

\section{Acknowledgments}

This work was supported by a research grant from the STEPI of 97-N1-02-04-A-09 (to O.-Y. Kwon). 
Burgi-Saville M. E., Gerber H., Peter H. J., Paulsson M., Aeschlimann D., Glaser C., Kaempf F., Ruchti C., Sidiropoulos I. and Burgi U. (1997), Expression patterns of extracellular matrix components in native and cultured normal human thyroid tissue and in human toxic adenoma tissue. Thyroid 7, 347-356.

Chang C., Lee H. J. and Lee Y. F. (1997), Identification of the human aldolase A gene as the first induced target for the TR2 orphan receptor, a member of the steroid hormone receptor superfamily. Biochem. Biophys. Res. Commun. 235, 205-211.

Diachenko L. B., Ledesma J., Chenchik A. A. and Siebert P. D. (1996), Combining the technique of RNA fingerprinting and differential display to obtain differentially expressed mRNA. Biochem. Biophys. Res. Commun. 219, $824-828$.

Giannoni P., Scarabelli L., Orunesu M. and Cesarone C. F. (1995), In vitro effect of 3,5,3'-triiodothyronine on poly(ADP-ribosyl)ation of DNA topoisomerase I. Italian J. Biochem. 44, 129-136.

Gomez P. F., Ito K., Huang Y., Otsu K., Kuzumaki T. and Ishikawa K. (1994), Dietary and hormonal regulation of aldolase B gene transcription in rat liver. Arch. Biochem. Biophys. 314, 307-314.

Kim P. S. and Arvan P. (1991), Folding and assembly of newly synthesized thyroglobulin occurs in a pre-golgi compartment. J. Biol. Chem. 256, 12412-12418.

Kim P. S. and Arvan P. (1993), Hormonal regulation of thyroglobulin export from the endoplasmic reticulum of cultured thyrocytes. J. Biol. Chem. 268, 4873-4879.

Kim P. S., Kwon O.-Y. and Arvan P. (1996), An endoplasmic reticulum storage disease causing congenital goiter with hypothyroidism. J. Cell Biol. 133, 517-527.

Kohn L. D. and Valente W. A. (1989), FRTL-5 manual: A Current Guide. In: FRTL-5 Today, (Perrild H. ed.). Elsevier Science Publishers, pp 243-262.
Kurkinen M., Condon M. R., Blumberg B., Barlow D. P., Quinones S., Saus J. and Pihlajaniemi T. (1987), Extensive homology between the carboxyl-terminal peptides of mouse alpha-1(IV) and alpha-2(IV) collagen. J. Biol. Chem. 262, 8496-8499.

Liang P. and Pardee A. (1992), Differential display of eukaryotic messenger RNA by of the polymerase chain reaction. Science 257, 967-970.

Liu H. C. and Towle H. C. (1994), Functional synergism between multiple thyroid hormone response elements regulates hepatic expression of the rat S14 gene. Mol. Endocrinol. 8, $1021-1037$.

Medeiros-Neto G., Targovnik H. M. and Vassart G. (1993), Defective thyroglobulin synthesis and secretion causing goiter and hypothyroidism. Endo. Rev. 14, $165-183$.

Mukai T., Joh K., Arai Y., Yatsuki H. and Hori K. (1986), Tissue-specific expression of rat aldolase A mRNAs: Three molecular species differing only in the 5'-terminal sequences. J. Biol. Chem. 261, 3347-3354.

Onishi Y. and Kizaki H. (1996), Molecular cloning of the genes suppressed in RVC lymphoma cells by topoisomerase inhibitors. Biochem. Biophys. Res. Commun. 228, 7-13.

Shibahara K., Asano M., Ishida Y., Aoki T., Koike T. and Honjo T. (1995), Isolation of novel mouse gene MA-3 that is induced upon programmed cell death. Gene 166, 297-301.

Ulisses S., Rucci N., Piersanti D., Carosa E., Graziano F. M., Pavan A., Ceddia P., Arizzi M., Muzi P., Cironi L., Gnessi L., D'Armiento M. and Jannini E. A. (1998), Regulation by thyroid hormone of the expression of basement membrane components in rat prepubertal sertoli cells. Endocrinology 139, 741-747. 